\title{
Colony morphotypes on Congo red agar segregate along species and drug susceptibility lines in the Mycobacterium avium-intracellulare complex
}

\author{
Gerard A. Cangelosi, Christine O. Palermo, Jean-Pierre Laurent, \\ April M. Hamlin and William $\mathrm{H}$. Brabant
}

Seattle Biomedical Research Institute, 4 Nickerson St, Seattle, WA 98109, USA
Author for correspondence: Gerard A. Cangelosi. Tel: +1206284 8846. Fax: +1 2062840313. e-mail : cangelos@u.washington.edu

\begin{abstract}
Isolates of the Mycobacterium avium-intracellulare complex (MAC) have long been known to segregate into transparent, opaque and rough colony morphotypes that differ from each other in clinically important parameters including drug susceptibility and virulence. Here the authors report additional morphotypic variation that occurs on two levels: interspecific (between $M$. avium and $\boldsymbol{M}$. intracel/ulare) and intraspecific (within individual $\boldsymbol{M}$. avium isolates). Clinical isolates of $\boldsymbol{M}$. avium grown on Congo red (CR) plates formed red, pink or mixed (red and white) opaque colonies, while $M$. intracellulare isolates formed purely white opaque colonies. A quantitative CR binding assay showed that this interspecific differential applies to transparent as well as opaque colony variants; however, it was less pronounced among laboratory reference strains than among recent clinical isolates. Opaque colonies of $\boldsymbol{M}$. avium isolates with 'mixed' phenotypes segregated into stable opaque red and white variants with shared IS1245 banding patterns (intraspecific segregation). White segregants of $M$. avium were more flocculent and significantly more resistant to ciprofloxacin and rifamycin drugs than were red segregants. Thus, cultivation on CR agar revealed a previously unknown multidrug resistant colony morphotype of $M$. avium.
\end{abstract}

Keywords: Mycobacterium avium-intracellulare complex (MAC), AIDS, phenotypic switching, antibiotic resistance, pre-rRNA

\section{INTRODUCTION}

The Mycobacterium avium-intracellulare complex (MAC) is responsible for common opportunistic infections of immunodeficient individuals, especially AIDS patients with low $\mathrm{CD}^{+}$cell counts. Most AIDSassociated MAC infections involve the species $M$. avium, while non-AIDS-associated infections involve $M$. avium and $M$. intracellulare at approximately equal frequencies. In contrast to the primary mycobacterial pathogens Mycobacterium tuberculosis and Mycobacterium leprae, which are transmitted from person to person, MAC infections are normally acquired from environmental sources (Guthertz et al., 1989; Grange,

Abbreviations: $C R$, Congo red; HMC, Harborview Medical Center; MAC, Mycobacterium avium-intracellulare complex; RCRB, relative Congo red binding.
1991; Inderlied et al., 1993; Peloquin, 1997; Swanson et al., 1998; Von Reyn et al., 1994).

Most MAC isolates segregate into smooth transparent $(\mathrm{SmT})$, smooth opaque ( $\mathrm{SmD}$ or $\mathrm{SmO}$ ) and sometimes rough colony type variants. Transparent variants predominate in fresh clinical isolates but opaque variants are usually also present. Opaque variants grow faster in vitro and usually predominate after several passages. Clones switch back and forth between opaque and transparent morphotypes, but conversion to the rough morphotype involves well-characterized chromosomal deletions and is irreversible. All three morphotypic variations are known or presumed to involve cell envelope structures (Inderlied et al., 1993; Belisle et al., 1993; Belisle \& Brennan, 1994; Prinzis et al., 1994).

The MAC is intrinsically resistant to most antibiotics used to treat tuberculosis and leprosy. Drugs such as 
clarithromycin, azithromycin, rifabutin, ethambutol, fluoroquinolones, clofazamine and amikacin, which are effective against primary isolates of the MAC, quickly lose effectiveness unless administered in multi-drug regimens. The intrinsic drug resistance of the MAC is usually ascribed to cell envelope impermeability, but other mechanisms have also been described (Inderlied $e t$ al., 1993; Mdluli et al., 1998). For unknown reasons, transparent variants are more multi-drug resistant than are opaque variants. Diagnostic drug susceptibility testing of MAC isolates is complicated by the occurrence of multiple variants with differing drug susceptibility phenotypes (Inderlied et al., 1993; Jarlier \& Nikaido, 1994; Portillo-Gomez et al., 1995; Peloquin, 1997; Heifets, 1996; Sison et al., 1996).

In light of the importance of cell envelopes to the pathogenesis, diagnosis and treatment of MAC infections, we asked whether a biological stain used to assess cell-surface variation in other bacteria could provide new information on cell-surface variation in the MAC. Congo red (CR) is a planar, hydrophobic, diazo dye that binds to lipids and lipoproteins, which are abundant in mycobacterial cell walls, and to a broad range of other macromolecules. CR has been used for histological detection of amyloid plaques associated with Alzheimer's disease (Sipe, 1994), and in the analysis of cell-surface variability in diverse bacterial pathogens (Andrews \& Maurelli, 1992; Bhaduri et al., 1997; Pace et al., 1997; Theisen, 1996; Tompkins et al., 1997; Ziebuhr et al., 1997). CR staining of MAC colonies revealed previously unseen variation on both the interspecific (between $M$. avium and $M$. intracellulare) and intraspecific (within individual M. avium isolates) levels. Intraspecific variation in $C R$ staining coincided with potentially significant variation in the susceptibility of the pathogens to antibiotics.

\section{METHODS}

Bacterial strains and culture media. Clinical isolates of the MAC (Table 1) came from the clinical laboratory at the Harborview Medical Center (HMC), Seattle, WA, USA (kind gifts from Carolyn Wallis and Marie Coyle). These strains had been grown on Middlebrook $7 \mathrm{H} 11$ agar plates either directly from specimen or from primary BACTEC broth cultures. Growth on these plates was determined to be the MAC by AccuProbe testing (Gen-Probe), then stored at $-70^{\circ} \mathrm{C}$. Thawed stocks were subcultured twice on $7 \mathrm{H} 11$ plates before inoculation onto Lowenstein-Jensen (LJ) slants for transport to our laboratory at the Seattle Biomedical Research Institute (total of four to five transfers from specimen). Reference strains (Table 2) were kindly provided on LJ slants by John Belisle, Colorado State University, or were obtained lyophilized from the American Type Culture Collection (ATCC). Species-specific AccuProbe tests (kind gifts from Jean-Paul Tousignant, Gen-Probe) were conducted at the HMC according to manufacturer's protocols.

Dubos-albumin medium (DANG) contained Dubos Broth Base and Dubos Medium Albumin enrichment (Difco Laboratories). Glycerol was added to $5 \%(\mathrm{v} / \mathrm{v})$ before autoclaving to make Dubos-albumin-glycerol (DAG) medium. Middlebrook-
Table 1. CR staining of opaque colonies of clinical isolates from the HMC

\begin{tabular}{|llcc|}
\hline Isolate* & \multicolumn{1}{c}{ CR } & $\begin{array}{c}\text { M. avium } \\
\text { probe }\end{array}$ & $\begin{array}{c}\text { M. intracellulare } \\
\text { probe }\end{array}$ \\
\hline HMC01 & Red & + & - \\
HMC02 & Red, pink, white & + & - \\
HMC04 & Red & + & - \\
HMC05 & Pink & + & - \\
HMC07 & Red, pink & + & - \\
HMC08 & Pink, white & + & - \\
HMC10 & Red, white & + & - \\
HMC11 & Pink & + & - \\
HMC12 & White & - & + \\
HMC13 & White & - & + \\
HMC14 & White & - & - \\
HMC15 & Red & + & - \\
HMC16 & Pink & + & - \\
HMC19 & Red, white & + & - \\
HMC20 & Pink & + & - \\
HMC22 & Red, white & + & - \\
HMC23 & Pink & + & + \\
HMC24 & Pink & + & - \\
HMC27 & White & - & - \\
\hline
\end{tabular}

*An additional isolate, HMC26, did not form opaque colonies.

albumin-glycerol medium (MAG) was Middlebrook 7H9 broth with ADC enrichment (Becton Dickinson Microbiology Systems), $0 \cdot 1 \%(\mathrm{w} / \mathrm{v})$ Casitone (Difco) and $5 \%(\mathrm{v} / \mathrm{v})$ glycerol. Tween 80 was added to $0.083 \%(\mathrm{w} / \mathrm{v})$ to make MAGT medium. Agar plates were solidified with $1.5 \%(\mathrm{w} / \mathrm{v})$ Difco agar. Stock solutions of CR (Sigma) were prepared in water and stored for no more than $60 \mathrm{~d}$ at room temperature. CR was added to media before autoclaving to a final concentration of $100 \mu \mathrm{g} \mathrm{ml}^{-1}$. Excessive autoclaving and exposure of CR media to UV light was avoided because they resulted in palecoloured plates and weak staining.

Relative Congo red binding (RCRB) assays. A CR binding assay (Andrews \& Maurelli, 1992) was adapted for use on the MAC as follows. Colonies picked from CR plates were suspended with vigorous vortexing in $3.5 \mathrm{ml}$ deionized water to $\mathrm{OD}_{660} 0 \cdot 1-1 \cdot 5$. After recording the $\mathrm{OD}_{660}$ of the suspension, cells were pelleted, resuspended in $200 \mu \mathrm{l}$ acetone, vortexed and allowed to sit at room temperature for $2 \mathrm{~h}$. Cells were then pelleted by high-speed microcentrifugation and $\mathrm{CR}$ in the supernatants was measured spectrophotometrically at $488 \mathrm{~nm}$. The RCRB value was defined as the $A_{488}$ of the acetone extracts divided by the $\mathrm{OD}_{660}$ of the original cell suspension.

IS1245 banding patterns. Insertion sequence IS1245-based RFLP analysis was performed as described by Garzelli $e t$ al. (1997) with the following modifications. Genomic DNA ( $3 \mu \mathrm{g})$ extracted from $M$. avium cells was digested to completion with $8 \mathrm{U} \mathrm{NruI}$ or $8 \mathrm{U}$ PvuII (New England Biolabs). After $0.7 \%$ agarose gel electrophoresis, DNA was partially hydrolysed and transferred onto Nytran membranes (Sambrook et al., 1989). IS1245 probe was prepared by incorporation of $\left.{ }^{32} \mathrm{P}\right] \mathrm{dCTP}$ during PCR from $M$. avium DNA using primers P1 
Table 2. CR staining of opaque colonies of reference strains

\begin{tabular}{|llcll|}
\hline Strain* & \multicolumn{1}{c}{ Species $\dagger$} & Serotype & Source $\neq$ & CR \\
\hline B-92 & M. avium & 1 & CSU & White \\
35717 & M. avium & 1 & ATCC & Pink \\
128 & M. avium & 3 & CSU & White \\
Germany & & & & \\
$13528-1079$ & M. avium & 4 & CSU & White \\
$6450-204$ & M. avium & 9 & CSU & Pink \\
$1602-1965$ & M. avium & 10 & CSU & Pink \\
$14186-1424$ & M. avium & 11 & CSU & Pink \\
2993 & M. avium & 21 & CSU & Pink \\
35713 & M. avium & Rough & ATCC & Red \\
25122 & M. intracellulare & 13 & CSU & White \\
P-39 & M. intracellulare & 14 & CSU & White \\
35848 & M. intracellulare & 15 & ATCC & White \\
Yandle & M. intracellulare & 16 & CSU & White \\
13950 & M. intracellulare & 16 & ATCC & White \\
P-54 & M. intracellulare & 17 & CSU & White \\
23393 & M. intracellulare & 23 & CSU & White \\
12645 & M. intracellulare & 24 & CSU & White \\
$72-888$ & M. intracellulare & 25 & CSU & White \\
6845 & M. intracellulare & 28 & CSU & White \\
25169 & M. intracellulare & Not known & ATCC & White \\
\hline
\end{tabular}

* Eight additional strains representing MAC serotypes 2, 5, 6, 12, $15,18,19$ and 20 did not form opaque colonies or produced yellow pigment that obscured CR results, and therefore are not included in the table.

†Per Frothingham \& Wilson (1993), Wayne et al. (1993) and the ATCC.

‡CSU, kind gift from J. Belisle, Colorado State University, USA.

(5'-GCC GCC GAA ACG ATC TAC-3') and P2 (5'-AGG TGG CGT CGA GGA AGA C-3'). The probe was hybridized to the blots in $20 \times$ SSC (Sambrook et al.,1989) for $12 \mathrm{~h}$ at $42{ }^{\circ} \mathrm{C}$. Membranes were washed sequentially in $2 \times$ SSC, $0.1 \%$ SDS at room temperature; $0.2 \times$ SSC, $0.1 \%$ SDS at room temperature; and $0.2 \times$ SSC, $0.1 \%$ SDS at $70^{\circ} \mathrm{C}$. Hybridization was detected by exposing the filters to a Molecular Dynamics standard phosphor screen for 2-48 h. Exposed phosphor screens were scanned in a Storm 860 PhosporImager using Molecular Dynamics ImageQuaNT software.

E-tests. Drug susceptibility testing of slow-growing mycobacteria using ciprofloxacin E-test strips (MIC range 0.002-32 $\mu \mathrm{g} \mathrm{ml}^{-1}$ ) has been described by Wanger \& Mills (1994). Colonies picked from CR plates were suspended in $1 \mathrm{ml} \mathrm{MAG}$ broth to $\mathrm{OD}_{660} \sim 0 \cdot 1$. Sterile cotton swabs were used to streak the suspension onto MAG, MAGT, DANG or DAG plates in a three-way pattern, and E-test strips (Remel Microbiology Products) were applied aseptically as prescribed by the manufacturer. Plates were incubated in polyethylene bags at $37^{\circ} \mathrm{C}$ under $5 \% \mathrm{CO}_{2}$ for $13-14 \mathrm{~d}$. Sharply defined zones of inhibition of opaque colony growth were usually visible after $7 \mathrm{~d}$ on surfactant-containing MAGT, DANG or DAG plates, or 13-14 d on surfactant-free MAG plates.

Pre-rRNA assays. The effects of rifampin and rifabutin on prerRNA pools in $M$. avium were measured as described previously (Cangelosi et al., 1996), with the following modi-

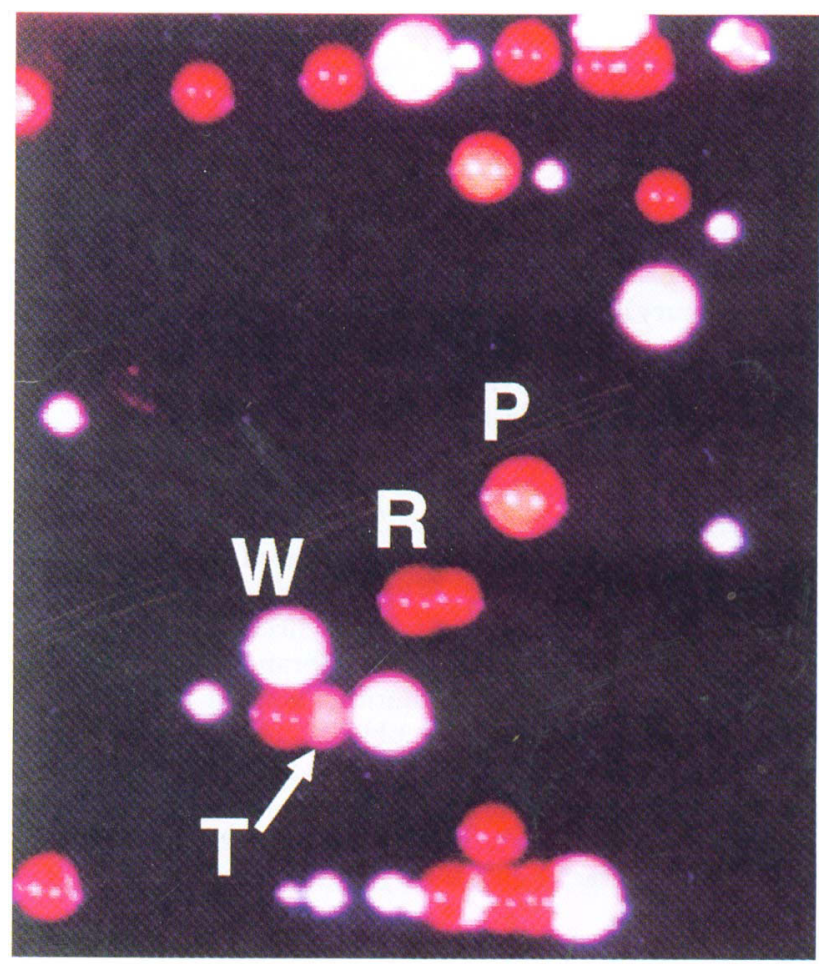

Fig. 1. Colonies of $M$. avium isolate $\mathrm{HMCO2}$, showing typical red (R), pink (P), white $(W)$ and transparent (T) colony morphotypes on DAG-CR agar.

fications. Colonies picked from $\mathrm{CR}$ plates were suspended in $6 \mathrm{ml} \mathrm{MAG}$ broth to an $\mathrm{OD}_{600} 0 \cdot 05-0 \cdot 10$. Each suspension was distributed to a series of autoclaved $15 \mathrm{ml}$ glass test tubes $(1 \mathrm{ml}$ per tube) pre-filled with rifampin (Sigma) to yield final concentrations of $0,0 \cdot 1,0.4,1.6,6.4$ and $25.6 \mu \mathrm{g} \mathrm{ml}^{-1}$, or rifabutin (kind gift from Pharmacia \& Upjohn) to yield final concentrations of $0,0 \cdot 1,0 \cdot 6,3.6$ and $21.6 \mu \mathrm{g} \mathrm{ml}^{-1}$. Tubes were incubated for $24 \pm 2 \mathrm{~h}$ on a rotary shaker at $37^{\circ} \mathrm{C}$ under $5 \%$ $\mathrm{CO}_{2}$. Cells were harvested and lysed using a lysozyme/ proteinase $\mathrm{K} /$ detergent/heat method as described previously (Cangelosi et al., 1996), except that Proclin 150 was omitted from the lysing solution. RNA was extracted by adding $100 \mu \mathrm{l}$ of each lysate to a $2 \mathrm{ml}$ microcentrifuge tube containing $350 \mu \mathrm{l}$ extraction buffer $(50 \mathrm{mM}$ Tris/ $\mathrm{HCl}, 10 \mathrm{mM}$ EDTA, $100 \mathrm{mM}$ $\mathrm{NaCl}$ and $5 \%$, w/v, SDS, $\mathrm{pH} 7 \cdot 6), 100 \mu \mathrm{l} 1$-methyl-2pyrrolidinone (Aldrich Chemical) and $550 \mu \mathrm{l} 70 \%$ phenol/ water/chloroform (Applied Biosystems). The mixture was vortexed, heated to $85^{\circ} \mathrm{C}$ for $12 \mathrm{~min}$, and centrifuged at $13000 \mathrm{~g}$ for $8 \mathrm{~min}$. The aqueous layer (about $400 \mu \mathrm{l}$ ) was transferred to a new tube to which was added $600 \mu \mathrm{l}$ phenol/water/chloroform. After vortexing and centrifugation at $13000 \mathrm{~g}$ for $5 \mathrm{~min}$, the aqueous layer (about $300 \mu \mathrm{l}$ ) was removed to a sterile $1.7 \mathrm{ml}$ microcentrifuge tube, to which was added 0.1 vol. $3 \mathrm{M}$ sodium acetate and $1 \mathrm{ml}$ ethanol. After precipitation at $-20^{\circ} \mathrm{C}$ for $1 \mathrm{~h}$, the tube was centrifuged as above for $15 \mathrm{~min}$. The pellet was air-dried for 5-10 min, then resuspended in $200 \mu \mathrm{l}$ TE buffer. The solution was applied to Nytran Plus membrane filters (Schleicher \& Schuell) using a slot-blot apparatus, and fixed by UV cross-linking (Cangelosi et al., 1996).

MAVT75, a riboprobe specific for the 5 ' leader region of the pre-16S rRNA of $M$. avium, was prepared and hybridized to 
slot blots as follows. PCR was used to amplify 180 bp genomic DNA immediately upstream of the $5^{\prime}$ terminus of the mature $16 \mathrm{~S}$ rRNA of $M$. avium ATCC 25291. The forward primer was L1 (5'-GGG TTG CCC CGA AGC G-3') and the reverse primer was MACPCR5 (5'-AAT TTA ATA CGA CTC ACT ATA GGG ACG CAG CGA GGT GAA TTT CAA ATC-3'). MACPCR5 has a 25-base linker containing the T7 RNA polymerase promoter and a spacer sequence. Transcripts complementary to pre-rRNA were generated from the purified amplification products with the incorporation of $\left[\alpha^{32} \mathrm{P}\right] \mathrm{UTP}$ by using T7 RNA polymerase. Labelled transcripts were purified and stored as described previously (Cangelosi et al., 1996, 1997). Slot-blot filters were pre-hybridized by shaking for $2 \mathrm{~h}$ at $50^{\circ} \mathrm{C}$ in a solution of $0.6 \mathrm{M} \mathrm{NaCl}, 90 \mathrm{mM}$ Tris $\mathrm{pH}$ 7.5, $10 \mathrm{mM}$ EDTA, $0.5 \%$ SDS, $30 \%$ purified formamide, $10 \mu \mathrm{g}$ ultrapure yeast tRNA $\mathrm{ml}^{-1}$, prepared in diethyl-pyrocarbonate-treated water. Probes were added to a final concentration of $1 \times 10^{6}$ c.p.m. ml ${ }^{-1}$, and hybridization proceeded overnight at $50{ }^{\circ} \mathrm{C}$ with shaking. Membranes were washed in successive changes of wash solution $(90 \mathrm{mM} \mathrm{NaCl}, 9 \mathrm{mM}$ Tris, $0.6 \mathrm{mM}$ EDTA, $1 \% \mathrm{SDS}, \mathrm{pH} 8.0$ ) for 1-2 min at room temperature, $1 \mathrm{~h}$ at $50^{\circ} \mathrm{C}$, and $30 \mathrm{~min}$ at $80^{\circ} \mathrm{C}$. The still-moist membranes were sealed in plastic wrap for phosphorimage analysis as described above. Phosporoimage signals were quantified using the Molecular Dynamics ImageQuaNT Peak Finder program.

\section{RESULTS}

\section{Species-level differences in CR staining}

Twenty clinical isolates of the MAC from the HMC, Seattle, were streaked from LJ slants onto DAG plates containing $100 \mu \mathrm{g} \mathrm{CR} \mathrm{ml} \mathrm{m}^{-1}$. Preliminary experiments had shown that this concentration of the stain inhibited growth slightly but resulted in vivid staining of colonies grown for $2-3$ weeks at $37{ }^{\circ} \mathrm{C}$ under $5 \% \mathrm{CO}_{2}$. Most isolates formed both opaque and transparent colonies distinguishable by stereoscopic microscopy with transmitting light (Belisle \& Brennan, 1994). One isolate formed transparent colonies only and a few isolates formed opaque colonies only. Opaque colonies exhibited considerable diversity with regard to CR staining (Table 1). Most isolates formed uniformly pink to deep red opaque colonies. Four isolates (HMC12, 13, 14 and 27) resisted the dye, appearing uniformly white on $C R$ plates. Six isolates formed heterogeneous red, white and/or pink colonies ('mixed' phenotype; Fig. 1). The HMC laboratory had determined that all 20 isolates were the MAC by using the MAC-specific AccuProbe test, but species identifications ( $M$. avium or $M$. intracellulare) were not known. To determine whether $\mathrm{CR}$ staining phenotypes differed between species, isolates were tested with $M$. avium-specific and $M$. intracellulare-specific AccuProbe kits. The four purely white isolates proved to be $M$. intracellulare, while the red, pink and mixed isolates were $M$. avium (Table 1).

The results in Table 1 suggested that CR staining may segregate along species lines in the MAC, with the capacity to form red, pink or mixed colonies confined to $M$. avium. To explore this possibility further, we examined a collection of 20 laboratory reference strains

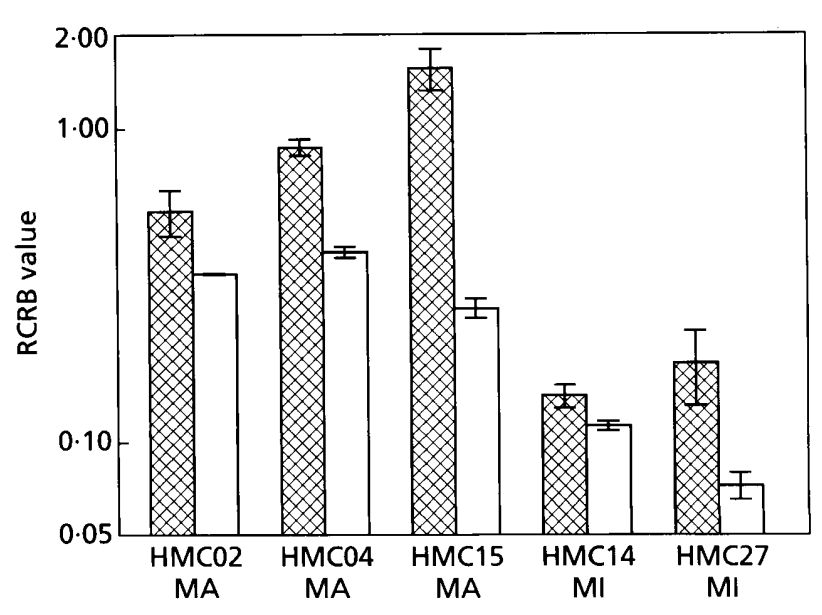

Fig. 2. CR staining of opaque $(\square)$ and transparent $(\square)$ colonies of $M$. avium (MA) and $M$. intracellulare (MI). The RCRB value is the $A_{488}$ of acetone extracts divided by $O D_{660}$ of cell suspensions before acetone extraction. Higher values correspond to more CR binding. Means and standard deviations of three experiments are shown.

of the MAC. This collection included $11 \mathrm{M}$. intracellulare strains and $9 \mathrm{M}$. avium strains, together representing 16 serotypes plus a rough mutant. As with HMC isolates of $M$. intracellulare, all reference strains of $M$. intracellulare resisted the stain (Table 2). Reference strains of $M$. avium stained more weakly as a group than did the HMC isolates, and some were indistinguishable from $M$. intracellulare. The rough mutant of $M$. avium stained dull red, indicating that cell-wall glycopeptidolipids, which are absent in rough variants (Belisle et al., 1993; Belisle \& Brennan, 1994), are not required for CR staining. The results in Tables 1 and 2 suggest that the red, pink and mixed phenotypes are confined to $M$. avium, while the white phenotype can be found in both species.

\section{Interspecies variation in CR staining of transparent colonies}

In contrast to the vivid heterogeneity in CR staining seen with opaque colonies, transparent colonies of all isolates appeared pale translucent pink (Fig. 1). Transparent colonies of $M$. intracellulare appeared slightly lighter than transparent colonies of $M$. avium, but the visual distinction was subtle at best. To determine whether or not there truly was a difference, we used a quantitative RCRB assay. Colonies were picked from CR plates and acetone-extractable CR was measured spectrophotometrically $\left(A_{488}\right)$ and normalized to cell density. Transparent colonies as well as opaque colonies of $M$. intracellulare stained less intensely in this assay than did their M. avium counterparts (Fig. 2). Therefore, among the five strains analysed in this way, interspecies variation in $\mathrm{CR}$ staining of the MAC applied to transparent as well as opaque variants. 
Morphotypic variation in the MAC

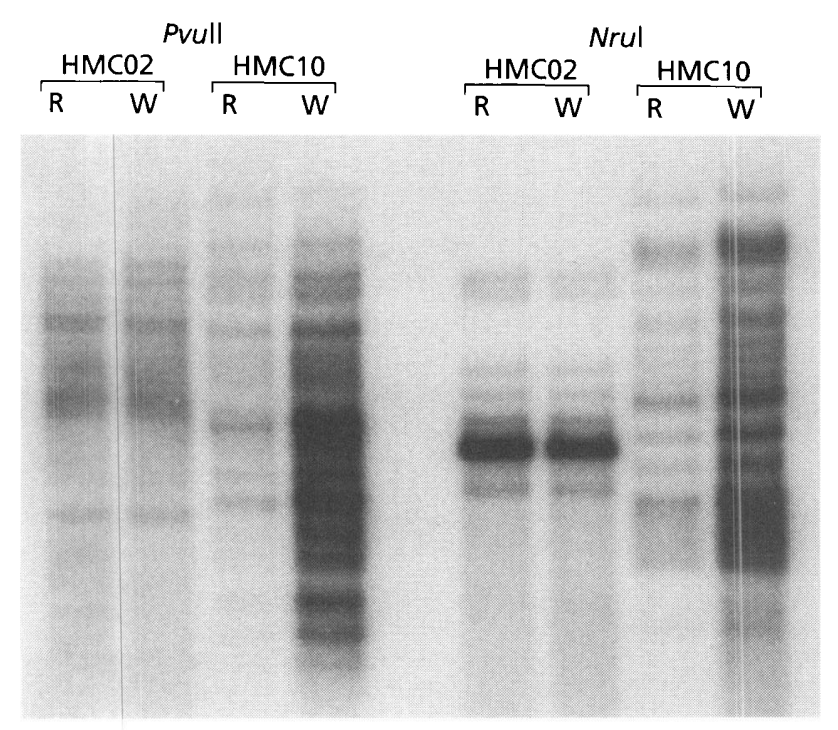

Fig. 3. 151245 banding patterns of restriction-endonucleasedigested genomic DNA from red (R) and white $(W)$ variants of $M$. avium isolates $\mathrm{HMCO2}$ and $\mathrm{HMC} 10$.

\section{Red and white segregants of 'mixed' $M$. avium isolates}

Two HMC isolates with 'mixed' phenotypes, HMC02 and HMC10, were studied further. Red and white opaque colonies were subcultured on CR plates to obtain stable, homogeneous red and white derivative clones. Although the two morphotypes were originally detected on DAG-CR plates, they were equally visible on MAG-CR (no Tween 80). They were indistinguishable on plates lacking CR, appearing as smooth domed opaque $(\mathrm{SmD})$ colonies. Growth rates of the two morphotypes were similar, as was the microscopic appearance of cells in acid-fast and Gram-stained smears. However, white variants picked from agar media and suspended in aqueous solutions, or grown in broth media, were more flocculent than were red variants. Because polyclonal infections are common in MAC disease (Inderlied et al., 1993), we used IS1245 mapping (Garzelli et al., 1997) to determine whether red and white variants of $\mathrm{HMCO} 2$ and $\mathrm{HMC} 10$ are separate strains or segregants of single strains. The results showed that they are segregants of single strains (Fig. 3).

\section{Drug resistance of white $M$. avium variants}

We asked whether CR red-white segregation coincided with differences in drug susceptibility, as is the case with opaque-transparent segregation (Inderlied et al., 1993; Belisle \& Brennan, 1994). Attempts to measure drug susceptibility using a broth-Alamar Blue assay (Collins \& Franzblau, 1997) were not successful. Although the Alamar Blue method works very well in our hands with stable MAC transparent strains and with $M$. tuberculosis, it gave variable results with opaque MAC variants, possibly because subpopulations of drug-resistant trans-

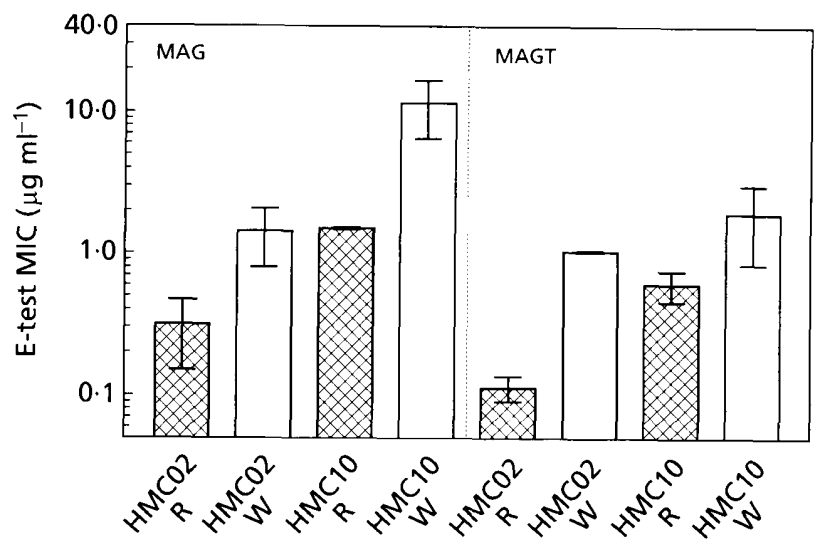

Fig. 4. Susceptibility of red (R) and white (W) segregants of $M$. avium isolates HMCO2 and HMC10 to ciprofloxacin E-test strips. Tests were conducted on MAG (no Tween 80) and MAGT (containing Tween 80) plates. MICs (E-test MIC) were read for opaque colony variants. Means and standard deviations of triplicate plates are shown.

parent variants were enriched to varying extents over the 7-10 d course of the test. Therefore, we used two tests that exclude the influence of drug-resistant transparent subpopulations.

The first method was agar diffusion using E-test strips (Wanger \& Mills, 1994). We chose the antimycobacterial drug ciprofloxacin for this analysis because it is available on E-test strips and is stable over the 2 weeks required to obtain results with the MAC. E-tests were conducted on MAGT plates (containing Tween 80) and MAG plates (no Tween 80). Opaque colonies formed well-defined zones of inhibition, usually with transparent colonies growing within. The presence of the surfactant Tween 80 resulted in sharper zones of inhibition and generally lower MICs, the latter being a well-known synergistic effect of surfactants with antibiotics (Inderlied et al., 1993; Heifets, 1996). With or without Tween 80 , white segregants exhibited higher MICs than did isogenic red segregants. Results of a typical experiment are shown in Fig. 4. In numerous repetitions of the experiment with varying inoculum sizes on MAG, MAGT, DAG or DANG (Dubos-glucose agar without glycerol), white segregants always had significantly higher MICs than did their red counterparts. The presence or absence of CR in the plates did not affect this outcome, although the presence of $C R$ resulted in slower growth and lower MICs overall.

The second approach was to measure the immediate effects of rifamycin-based drugs on rRNA precursor (pre-rRNA) pools in red and white opaque colonies. Rifamycins rapidly halt pre-rRNA synthesis while allowing rRNA processing to proceed, thereby draining pre-rRNA pools in susceptible, but not resistant, bacterial cells (Cangelosi et al., 1996; Britschgi \& Cangelosi, 1995). We conducted the test on isolated opaque colonies picked from CR plates and read results $24 \mathrm{~h}$ later, before 


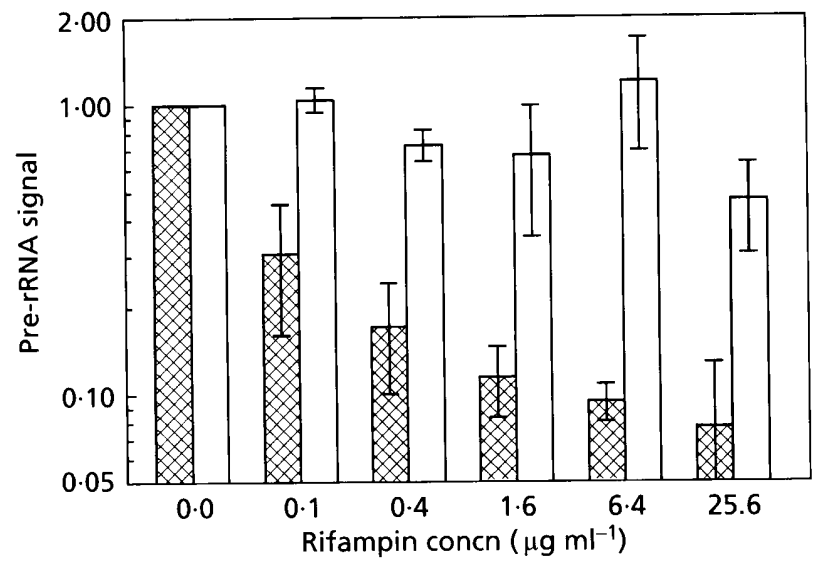

Fig. 5. Results of a typical pre-rRNA test for susceptibility to rifampin. Red $(\square)$ and white $(\square)$ segregants of $M$. avium isolate $\mathrm{HMCO2}$ were exposed in triplicate to dilution series of rifampin $\left(0-25.6 \mu \mathrm{g} \mathrm{ml}^{-1}\right)$. After $24 \mathrm{~h}$ incubation, pre-rRNA pools in each $1 \mathrm{ml}$ culture were measured by slot-blot hybridization and phosphorimage analysis as described in Methods. Phosphorimage signals were normalized to the no-drug control tube $\left(0 \mu \mathrm{g} \mathrm{ml}^{-1}\right)$ in each series. Means and standard deviations of the triplicate series are shown.

Table 3. Effects of rifamycin derivatives on pre-rRNA pools in red and white segregants of $M$. avium isolates $\mathrm{HMCO} 2$ and $\mathrm{HMC10}$

\begin{tabular}{|c|c|c|c|}
\hline \multirow[t]{2}{*}{ Isolate } & \multirow[t]{2}{*}{ Drug } & \multicolumn{2}{|c|}{$\begin{array}{l}\text { Relative inhibitory } \\
\text { concn }\left(\mu \mathrm{g} \mathrm{ml}^{-1}\right)^{*}\end{array}$} \\
\hline & & Red & White \\
\hline HMC02 & Rifampin & $1 \cdot 6$ & $>25.6$ \\
\hline $\mathrm{HMCO} 2$ & Rifabutin & 0.6 & 3.6 \\
\hline HMC10 & Rifabutin & $3 \cdot 6$ & $21 \cdot 6$ \\
\hline
\end{tabular}

* Results of triplicate experiments. Relative inhibitory concentration is arbitrarily defined as the minimum concentration needed to drain pre-rRNA pools by a factor of five relative to nodrug controls in all experiments; e.g. the relative inhibitory concentration of rifampin for the red variant in Fig. 5 is $1.6 \mu \mathrm{g} \mathrm{ml}^{-1}$.

drug-resistant transparent subpopulations could overgrow the cultures. White segregants were significantly more resistant than their red counterparts to both rifampin and rifabutin in this assay (Fig. 5 and Table 3 ). Pre-rRNA tests were conducted in surfactant-free MAG broth, and the presence or absence of surfactants in the plates from which colonies were picked did not affect outcomes.

To test the reproducibility of these observations, we returned to the original stock of HMC02 and repeated the entire process of growing 'mixed' colonies on DAG$\mathrm{CR}$, isolating red and white segregants, determining IS1245 banding patterns, and testing ciprofloxacin and rifamycin resistance; all had the same outcomes as shown in Figs 3-5.

\section{DISCUSSION}

CR staining phenotypes segregated along species lines within the MAC, and some M. avium isolates segregated into red-staining and white variants. These two segregation phenomena resembled each other in their manifestations on CR plates, but they differed from each other with regard to drug susceptibility: M. intracellulare is not more drug-resistant than $M$. avium generally (Inderlied et al., 1993; Peloquin, 1997; Heifets, 1996), and opaque white colonies of $M$. intracellulare isolate HMC14 were not more ciprofloxacin-resistant than opaque red colonies of M. avium isolates HMC04, HMC15 and HMC02 in E-tests (data not shown). Therefore, we will discuss interspecific and intraspecific segregation sequentially as separate (but possibly overlapping) phenomena.

If confirmed with a larger number of strains, segregation of CR staining phenotypes along species lines could form the basis for an inexpensive diagnostic method. Nucleic acid probes with separate specificity for $M$. avium and $M$. intracellulare are available to clinical laboratories (Gen-Probe); however, for reasons of cost many laboratories (including $\mathrm{HMC}$ ) use only GenProbe's unified MAC probe, leaving the question of species identity unanswered. Plating on CR agar could add species identity information for very little added cost. Our current data suggest that red-, pink- or mixedstaining isolates are always $M$. avium, but the observation of purely white colonies on DAG would have to be considered inconclusive. It takes $2-3$ weeks for $\mathrm{MAC}$ isolates to form colonies large enough to visually discern CR staining results; however, it may be possible to shorten this time frame using microscopy or the RCRB assay.

Because the HMC isolates of M. avium (Table 1) stained more intensely as a group than did laboratory reference strains of $M$. avium (Table 2), there was a clearer distinction between $M$. avium and $M$. intracellulare within the HMC group. The two populations were too diverse in their origins for easy explanation of this difference. It is very likely that the reference strains were maintained and transferred outside of animal hosts more extensively than the HMC isolates, which had been transferred no more than five times from specimen to CR plate. Therefore, the characteristic of deep-red staining may be lost from $M$. avium clones during adaptation to extracellular growth.

White opaque segregants of $M$. avium isolates were more resistant to ciprofloxacin and rifamycin drugs and also significantly more flocculent than their red-staining counterparts. If the white morphotype occurs in patients, its drug resistance could affect clinical outcomes. If, on the other hand, the white morphotype is an adaptation to in vitro growth, its drug resistance could be a manifestation of more general resistance to en- 
vironmental challenges (and to $\mathrm{CR}$ staining). MAC strains survive well in the environment and are resistant to disinfectants (Grange, 1991; Inderlied et al., 1993; Peloquin, 1997; Von Reyn et al., 1994). The flocculent, adherent white morphotype of $M$. avium may play a role in this toughness. It is interesting that the red morphotype predominated among recent clinical isolates of M. avium despite greater susceptibility to certain antibiotics. Red variants may be more readily ingested and disseminated in macrophages than flocculent white variants, rendering the former more pathogenic.

CR is a planar, hydrophobic molecule capable of binding diverse lipids and lipoproteins, which are abundant in mycobacterial cell walls. If CR binds to multiple sites on MAC cell envelopes, then the white phenotype may occur when the dye is excluded from these sites by unidentified cell-surface components. Consistent with this model, we identified several culture conditions that weaken or abolish the white phenotype in M. avium and $M$. intracellulare, resulting in red colonies. These include alternative carbon-energy sources, inhibition of mycolic acid synthesis by subinhibitory concentrations of isoniazid (Mdluli et al., 1998), incubation in mineral salts buffers, and other suboptimal conditions. In contrast, we have yet to identify conditions that perturb the red phenotype (unpublished data). These observations are consistent with a model wherein 'red' is the underlying phenotype of the MAC upon which 'white' is variably superimposed. Serotype-specific glycopeptidolipids, which are absent in rough mutants of M. avium (Belisle \& Brennan, 1994; Belisle et al., 1993), could in theory mask underlying CR binding sites. However, serotypes have not been reported to segregate within individual $M$. avium isolates with the high frequency of CR staining phenotype. MAC cells produce a variety of other surface-exposed and extracellular materials which could exclude CR (Frchcl et al., 1988; Lemassu et al., 1996). We are currently analysing the cell-surface lipid, protein and polysaccharide compositions of red and white segregants of $M$. avium.

Many of the factors that make MAC disease difficult to manage (intrinsic drug and disinfectant resistance, diagnostic challenges and frequent treatment failure) are ascribed to cell-surface variability, including the wellknown opaque-transparent-rough morphotypic switching phenomena. CR staining of MAC colonies revealed additional morphotypic variability that may also contribute to these factors. The ability to assess CR staining qualitatively on CR plates as well as quantitatively in the RCRB assay should help make it possible to characterize this potentially important phenomenon.

\section{ACKNOWLEDGEMENTS}

We thank Carolyn Wallis, Marie Coyle and John Belisle for providing MAC isolates and for their scientific input. David Sherman, Luiz Bermudez and Clark Inderlied also provided valuable insights. This work was supported by grants from the M. J. Murdock Charitable Trust and the National Institutes of Health (RO3AI41415 and R41AI40719). C.O.P. was supported by fellowships from the Mary Gates Endowment for Undergraduate Research and the NASA Space Grant Consortium. J.-P.L. was supported by NIH Training Grant no. T32AI07509.

\section{REFERENCES}

Andrews, G. P. \& Maurelli, A. T. (1992). $m x i$ A of Shigella flexneri 2a, which facilitates export of invasion plasmid antigens, encodes a homolog of the low-calcium response protein, LcrD, of Yersinia pestis. Infect Immun 60, 3287-3295.

Belisle, J. T. \& Brennan, P. J. (1994). Molecular basis of colony morphology in Mycobacterium avium. Res Microbiol $\mathbf{1 4 5}$, 237-242.

Belisle, J. T., McNeil, M. R., Chatterjee, D., Inamine, J. M. \& Brennan, P. J. (1993). Expression of the core lipopeptide of the glycolipid surface antigens in rough mutants of Mycobacterium avium. J Biol Chem 268, 10510-10516.

Bhaduri, S., Cottrell, B. \& Pickard, A. R. (1997). Use of a single procedure for selective enrichment, isolation, and identification of plasmid-bearing virulent Yersinia enterocolitica of various serotypes from pork samples. Appl Environ Microbiol 63, 1657-1660.

Britschgi, T. B. \& Cangelosi, G. A. (1995). Detection of rifampinresistant bacteria using DNA probes for precursor rRNA. Mol Cell Probes 9, 19-24.

Cangelosi, G. A., Brabant, W. H., Britschgi, T. B. \& Wallis, C. K. (1996). Detection of rifampin- and ciprofloxacin-resistant $M y c O-$ bacterium tuberculosis by using species-specific assays for precursor rRNA. Antimicrob Agents Chemother 40, 1790-1795.

Cangelosi, G. A., Hamlin, A. M., Buck, K. R. \& Scholin, C. A. (1997). Detection of stable pre-rRNA in toxigenic Pseudonitzschia species. Appl Environ Microbiol 63, 4859-4865.

Collins, L. \& Franzblau, S. G. (1997). Microplate alamar blue assay versus BACTEC 460 system for high-throughput screening of compounds against Mycobacterium tuberculosis and Mycobacterium avium. Antimicrob Agents Chemother 41, 1004-1009.

Frehel, C., Rastogi, N., Benichou, J.-C. \& Ryter, A. (1988). Do test tube grown pathogenic mycobacteria possess a protective capsule? FEMS Microbiol Lett 56, 225-230.

Frothingham, R. \& Wilson, K. H. (1993). Sequence-based differentiation of strains in the Mycobacterium avium complex. $J$ Bacteriol 175, 2818-2825.

Garzelli, C., Lari, N., Nguon, B., Cavallini, M., Pistello, M. \& Falcone, G. (1997). Comparison of three restriction endonucleases in IS1245-based RFLP typing of M. avium. J Med Microbiol 46, 933-939.

Grange, J. M. (1991). Environmental mycobacteria and human disease. Lepr Rev 62, 353-361.

Guthertz, L. S., Damsker, B., Bottone, E. J., Ford, E. G., Thaddeus, M. F. \& Janda, M. J. (1989). Mycobacterium avium and Mycobacterium intracellulare infection in patients with and without AIDS. J Infect Dis 160, 1037-1041.

Heifets, L. (1996). Susceptibility testing of Mycobacterium avium complex isolates. Antimicrob Agents Chemother 40, 1759-1767.

Inderlied, C. B., Kemper, C. A. \& Bermudez, L. E. M. (1993). The Mycobacterium avium complex. Clin Microbiol Rev 6, 266-310.

Jarlier, V. \& Nikaido, H. (1994). Mycobacterial cell wall: structure and role in natural resistance to antibiotics. FEMS Microbiol Lett 123, 11-18.

Lemassu, A., Ortalo-Magné, A., Bardou, F., Silve, G., Lanéelle, M.-A. \& Daffé, M. (1996). Extracellular and surface-exposed 
polysaccharides of non-tuberculous mycobacteria. Microbiology 142, 1513-1520.

Mdluli, K., Swanson, J., Fischer, E., Lee, R. E. \& Barry, C. E. (1998). Mechanisms involved in the intrinsic isoniazid resistance of Mycobacterium avium. Mol Microbiol 27, 1223-1233.

Pace, J. L., Chai, T., Rossi, H. A. \& Jiang, X. (1997). Effect of bile in Vibrio parahaemolyticus. Appl Environ Microbiol 63, 2372-2377.

Peloquin, C. A. (1997). Mycobacterium avium complex infection: pharmacokinetic and pharmacodynamic considerations that may improve clinical outcomes. Clin Pharmacokinet 32, 132-144.

Portillo-Gomez, L., Nair, J., Rouse, D. A. \& Morris, S. L. (1995). The absence of genetic markers for streptomycin and rifampicin resistance in Mycobacterium avium complex strains. $J$ Antimicrob Chemother 36, 1049-1053.

Prinzis, S., Rivoire, B. \& Brennan, P. J. (1994). Search for the molecular basis of morphological variation in Mycobacterium avium. Infect Immun 62, 1946-1951.

Sambrook, J., Fritsch, E. F. \& Maniatis, T. (1989). Molecular Cloning: a Laboratory Manual, 2nd edn. Cold Spring Harbor, NY : Cold Spring Harbor Laboratory.

Sipe, J. D. (1994). Amyloidosis. Crit Rev Clin Lab Sci 31, 325-354. Sison, J. P., Yao, Y., Kemper, C. A., Hamilton, J. R., Brummer, E., Stevens, D. A. \& Deresinski, S. C. (1996). Treatment of Mycobacterium avium complex infection: do the results of the in vitro susceptibility tests predict therapeutic outcome in humans? J Infect Dis 173, 677-683.

Swanson, D. S., Pan, X., Kline, M. W. \& 7 other authors (1998). Genetic diversity among Mycobacterium avium complex strains recovered from children with and without human immunodeficiency virus infection. $J$ Infect Dis 178, 776-782.

Theisen, M. (1996). Molecular cloning and characterization of $n l p H$, encoding a novel, surface-exposed, polymorphic, plasmidencoded 33 kilodalton lipoprotein of Borrelia afzelii. J Bacteriol 178, 6435-6442.

Tompkins, G. R., Wood, D. P. \& Birchmeier, K. P. (1997). Detection and comparison of specific hemin binding by Porphyromonas gingivalis and Prevotella intermedia. J Bacteriol 179, 620-626.

Von Reyn, C. F., Maslow, J. N., Barber, T. W., Falkinham, J. O. \& Arbeit, R. D. (1994). Persistent colonisation of potable water as a source of Mycobacterium avium infection in AIDS. Lancet 343, 1137-1141.

Wanger, A. \& Mills, K. (1994). E-test for susceptibility testing of Mycobacterium tuberculosis and Mycobacterium aviumintracellulare. Diagn Microbiol Infect Dis 19, 179-181.

Wayne, L. G., Good, R. C., Tsang, A. \& 13 other authors (1993). Serovar determination and molecular taxonomic correlation in Mycobacterium avium, Mycobacterium intracellulare, and $\mathrm{MycO}-$ bacterium scrofulaceum: a cooperative study of the International Working Group on Mycobacterial Taxonomy. Int J Syst Bacteriol 43, 482-489.

Ziebuhr, W., Heilmann, C. \& Gotz, F. (1997). Detection of the intercellular adhesion gene cluster (ica) and phase variation in Staphylococcus epidermidis blood culture strains and mucosal isolates. Infect Immun 65, 890-896.

Received 12 January 1999; revised 3 March 1999; accepted 10 March 1999. 